\title{
Prevalência de sinais e sintomas, fatores sociodemográficos associados e atitude frente aos sintomas em um centro urbano no Sul do Brasil
}

\author{
Raúl Mendoza-Sassi,${ }^{1}$ Jorge U. Béria, ${ }^{2}$ Nádia Fiori ${ }^{3}$ e Angéli Bortolotto ${ }^{3}$
}

Como citar Mendoza-Sassi R, Béria JU, Fiori N, Bortolotto A. Prevalência de sinais e sintomas, fatores sociodemográficos associados e atitude frente aos sintomas em um centro urbano no Sul do Brasil. Rev Panam Salud Publica. 2006;20(1):22-8.

RESUMO Objetivo. Determinar a prevalência de sinais e sintomas na população adulta, os fatores sociodemográficos associados e a atitude diante desses sintomas segundo o sexo.

Métodos. Um estudo transversal de base populacional foi realizado na Cidade de Rio Grande, Estado do Rio Grande do Sul, no ano de 2000. Foram entrevistadas 1259 pessoas com 15 anos ou mais. Aplicou-se um questionário estruturado contendo 18 sintomas, além de questões sociodemográficas. Foram calculadas a prevalência dos sintomas e as razões de prevalência para sexo, idade e classe econômica ajustados entre si. Também foi analisada a atitude tomada frente aos sintomas segundo o sexo.

Resultados. A idade média foi de 40,33 anos (53,9\% mulheres). O problema mais prevalente foi dor de cabeça $(55,4 \%)$. Dor nas juntas, insônia, prisão de ventre, pressão alta e falta de ar aumentaram com a idade. Tiveram maior prevalência em classes mais baixas: dor de cabeça, nervosismo, dor nas juntas e nas costas, insônia e depressão, tosse, pressão alta, dor torácica e falta de ar. Foram relatados 4424 problemas de saúde $(3,25$ por pessoa) e 60,2\% não geraram nenhuma atitude, 31,6\% levaram à automedicação e 8,2\% geraram procura por serviços médicos. Dor de cabeça, nervosismo, dor nas juntas e nas costas, insônia, depressão, prisão de ventre, pressão alta, dor no peito e falta de ar foram significativamente mais prevalentes nas mulheres, enquanto tosse foi significativamente maior entre os homens. As atitudes tomadas frente aos sinais e sintomas foram semelhante entre homens e mulheres.

Conclusões. É necessário estabelecer políticas de saúde que enfatizem a saúde mental e a educação para o auto-cuidado, salientando quais sintomas indicam a necessidade de procurar um serviço de saúde. Também é preciso estudar o papel da classe social na determinação do comportamento em saúde e da procura de cuidados.

Palavras-chave Classe social, comportamento na procura de cuidados médicos, prevalência, sinais e sintomas, Brasil.

1 Fundação Universidade Federal do Rio Grande (FURG), Departamento de Medicina Interna, Rio Grande (RS), Brasil. Enviar correspondência para este autor no seguinte endereço: Rua Dr. Pedro Armando Gatti 99, CEP 96260-080, Rio Grande, RS, Brasil. E-mail: dmiraul@furg.br
2 Universidade Luterana do Brasil (ULBRA), Faculdade de Medicina e Pós-Graduação em Saúde Coletiva, Canoas (RS), Brasil.

FURG, Faculdade de Medicina.
O estudo da morbidade autorelatada é útil para avaliar as condições de saúde da população. Vários estudos mostram a relação entre essa e outras medidas de morbidade ou mor- 
talidade $(1,2)$, sendo o auto-relato de sintomas ou problemas de saúde uma técnica bastante utilizada (3). Além disso, o estudo da carga de morbidade auto-relatada em nível populacional revela o que as pessoas percebem como necessidade, alargando a perspectiva que privilegia as avaliações feitas por serviços de saúde. No Brasil, embora vários estudos tratem da carga de morbidade referida em nível populacional, em termos de autoavaliação do estado de saúde, número de sintomas ou presença de doenças crônicas (4-6), poucos enfocam a prevalência dos tipos de sintomas referidos pela população, a associação desses sintomas com fatores demográficos e socioeconômicos e a atitude que os indivíduos tomam diante desses problemas.

Dessa forma, o presente estudo teve como objetivo descrever quais são os sintomas ou problemas de saúde referidos pela população adulta em um centro urbano no Sul do Brasil, como são afetadas as taxas de prevalência desses sintomas pela idade, o sexo e a classe socioeconômica e que atitude os indivíduos de cada sexo costumam tomar diante desses sintomas.

\section{MATERIAIS E MÉTODOS}

O estudo foi do tipo transversal, de base populacional, apropriado para os objetivos estipulados. A coleta de dados foi realizada no domicílio entre janeiro e maio de 2000 a partir de uma amostra probabilística das pessoas com 15 anos ou mais residentes no Município de Rio Grande, zona sul do Estado do Rio Grande do Sul. O Município tinha, no ano de 2000, 186544 residentes. Desses, 138478 tinham idade $\geq 15$ anos. As mulheres perfaziam 52\%. O grupo com idade de 15 a 24 anos representava $24 \%$, o grupo de 25 a 44 anos correspondia a $38,6 \%$, o grupo de 45 a 64 anos representava $26,7 \%$ e o grupo com 65 anos ou mais correspondia a 10,8\% da população. Somente 5\% residiam em zona rural (7).

O tamanho da amostra foi calculado para detectar uma prevalência esti- mada de sintomas de $60 \%$ na população, com precisão de $3 \%$ e nível de confiança de 95\% (IC95\%). Com esses parâmetros, o tamanho da amostra, já acrescido de $10 \%$ para perdas, foi calculado em 1125 pessoas.

A amostragem foi de tipo multiestágio, onde, em primeiro lugar, foram sorteados 45 dos 252 setores censitários existentes (amostragem por conglomerado). A seguir foi sorteada uma quadra e, imediatamente, uma esquina (amostragem simples). A partir da esquina selecionada, foi visitado um de cada três domicílios (amostragem sistemática) até serem visitados 12 domicílios por setor censitário. Dessa forma, foram visitadas 1348 pessoas, das quais 1259 responderam o questionário.

Todas as pessoas residentes na casa e elegíveis para o estudo (idade $\geq 15$ anos e capazes de responder às questões) eram entrevistadas. Um grupo de entrevistadores, treinados especificamente para este estudo, visitaram as casas selecionadas e aplicaram um questionário com questões demográficas e socioeconômicas e perguntas sobre a ocorrência de sinais e sintomas nos 2 meses anteriores. A idade, para fins de análise, foi agrupada em quatro faixas: 15 a 24 anos, 25 a 44 anos, 45 a 64 anos e 65 ou mais. A classificação econômica seguiu os critérios da Associação Brasileira de Pesquisas de Mercado (ABIPEME) (8). Para as análises, criaram-se três categorias: A/B, C e D/E. A presença de sinais e sintomas era identificada a partir de uma lista fechada com 18 itens, construída a partir de outros estudos já realizados (9). Para cada problema de saúde, perguntava-se se alguma providência tinha sido tomada. Em caso positivo, perguntava-se se o indivíduo tinha se automedicado ou se tinha procurado cuidado médico. Como automedicação foi considerada qualquer intervenção visando a desaparição ou diminuição do problema de saúde, incluindo portanto o uso de medicamentos sem prescrição ou com prescrição antiga, medicação caseira e fitoterapia, entre outros. Ao se investigar se a pessoa procurou ajuda para resolver o problema, não foi feita diferenciação entre sistema de saúde convencional ou medicina alternativa.

Os dados coletados foram digitados em um banco específico construído no programa Epi Info versão 6.04. Após dupla digitação independente, os dados foram corrigidos em termos de erros de amplitude e consistência. Logo a seguir foram traduzidos para o programa Stata 6.0. A análise estatística consistiu no cálculo das taxas de prevalência para as variáveis categóricas e no cálculo das médias e desvio padrão para as variáveis contínuas.

Para analisar os efeitos independentes das variáveis demográficas e socioeconômicas coletadas, foi utilizada a regressão de Poisson. Para cada sintoma ou problema referido, foram calculados as razões de prevalência (RP) e os respectivos IC95\% para as variáveis sexo, grupo de idade e classe econômica, ajustadas entre si e levando em consideração a amostragem por conglomerado. O teste estatístico utilizado no caso das variáveis dicotômicas foi o teste de Wald. Nas variáveis categóricas, onde os valores de RP mostrassem um ordenamento, foi realizado o teste de tendência linear. Nas variáveis categóricas sem ordenamento foi realizado o teste de Wald para provar a hipótese de que as RP obtidas para cada categoria analisada não eram diferentes da unidade. No que se refere ao comportamento frente aos sintomas, estudaram-se as diferenças entre os sexos, para entender melhor de que forma se diferenciavam homens e mulheres quando confrontados com os mesmos problemas de saúde. Para essa análise utilizou-se a prova do qui-quadrado $\left(\chi^{2}\right)$. Nas situações em que o número de casos esperados não satisfizesse os critérios para esse teste foi utilizado o teste exato de Fisher. O ponto de corte adotado para significância estatística foi $P$ $<0,05$.

\section{RESULTADOS}

Foram entrevistadas 1259 de 1348 pessoas possíveis, determinando uma 
perda de $7 \%$. A idade média do grupo foi de 40,33 anos $( \pm 7,71)$ e variou de 15 a 94 anos. A distribuição por idade foi a seguinte: $23,9 \%$ na faixa de 15 a 24 anos; $37,1 \%$ entre 25 e 44 anos; $27,8 \%$ entre 45 e 64 anos; e $11,2 \%$ com 65 anos ou mais. Houve uma predominância de mulheres $(53,9 \%)$ e de pessoas brancas $(84,7 \%)$. A média de anos de estudo foi $6,62( \pm 3,66)$. A classificação econômica da ABIPEME teve a seguinte distribuição: $26,9 \%$ nas classes A e B, 39,6\% na classe C e 33,5\% nas classes D e E. A renda média per capita (renda familiar mensal dividida pelo número de pessoas na família) foi de US\$168,32 ( $\pm 225,03)$. Quanto aos sinais e sintomas, $62,7 \%$ mencionaram ao menos um sintoma nos 2 meses anteriores à entrevista e $28,7 \%$ visitaram o médico no mesmo período.

Conforme se pode observar na tabela 1, o problema de saúde mais prevalente entre as pessoas entrevistadas foi a dor de cabeça, sendo que mais da metade dos entrevistados teve essa queixa. Na mesma tabela pode ser observado o efeito independente do sexo, grupo de idade e classe econômica sobre cada um dos sintomas referidos. Em termos gerais, observa-se que as mulheres apresentaram RP maiores para os sintomas pesquisados, com exceção apenas da tosse. Não houve diferença entre os sexos em relação a quatro sintomas: dor de barriga, dor de garganta, sangue na urina e sangue nas fezes.

TABELA 1. Prevalência e razões de prevalência de sintomas em indivíduos com 15 anos ou mais conforme sexo, idade e classe econômica, Rio Grande (RS), Brasil, 2000

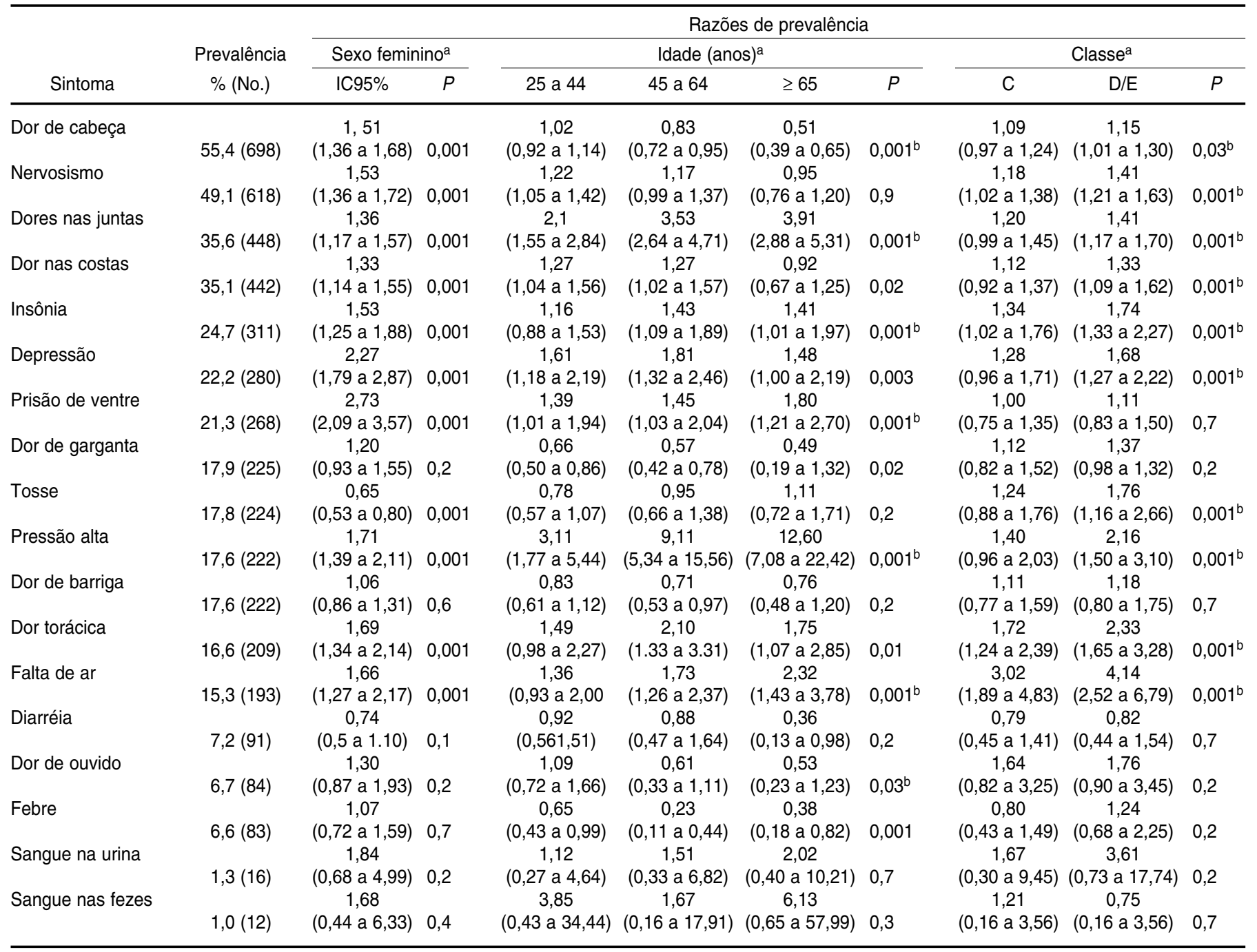

a Categorias de referência: sexo masculino, idade de 15 a 24 anos, classe A/B ABIPEME (8).

b Teste de tendência linear. 
Com respeito à idade, dor nas juntas, insônia, prisão de ventre, pressão alta e falta de ar aumentaram conforme aumentou a idade. Por outro lado, dor de cabeça, dor de garganta, diarréia, febre e dor de ouvido diminuíram com o aumento da idade. As diferenças não foram significativas para os problemas nervosismo, tosse, sangue na urina e sangue nas fezes (tabela 1).

A tabela 1 mostra também que 10 dos problemas referidos pelos entrevistados tiveram maior prevalência em classes econômicas mais baixas, mostrando uma relação linear: dor de cabeça, nervosismo, dor nas juntas e dor nas costas, insônia e depressão, tosse, pressão alta, dor torácica e falta de ar. Não tiveram associação significativa prisão de ventre, dor de garganta, dor de barriga, diarréia, dor de ouvido, febre, sangue na urina e sangue nas fezes.

A tabela 2 descreve as atitudes dos entrevistados frente aos problemas de saúde relatados. Foram relatados 4424 problemas de saúde, ou seja, uma média de 3,25 problemas de saúde por pessoa. Desse total, 60,2\% (2 664) não produziram nenhuma atitude, 31,6\% (1 399) levaram à automedicação e apenas 8,2\% (361) geraram procura por serviços médicos.

Além disso, a tabela 2 mostra que sintomas como dor de cabeça, nervosismo, dor nas juntas, dor nas costas, insônia, depressão, prisão de ventre, pressão alta, dor no peito e falta de ar foram significativamente mais prevalentes nas mulheres. Apenas a tosse foi significativamente maior entre os homens.

Entre os problemas que não geraram nenhum tipo de atitude, a insônia, a depressão e o nervosismo foram os mais freqüentes. A automedicação foi mais freqüente para dor de cabeça, prisão de ventre, febre e dor de garganta. $\mathrm{O}$ problema que mais gerou consulta foi a presença de sangue na urina (50\% das pessoas que apresentaram esse problema), mostrando a importância desse sintoma para a população estudada (tabela 2).

Finalmente, a tabela 2 indica que o comportamento em termos da tomada de atitudes frente aos sinais e sintomas foi muito semelhante entre homens e mulheres. Houve diferenças significa- tivas em termos de nervosismo, diante do qual uma maior proporção de homens não fez nada, enquanto as mulheres tenderam a se automedicar mais e a consultar mais. O mesmo ocorreu com a insônia, onde $95 \%$ dos homens decidiram não fazer nada, enquanto $17,5 \%$ das mulheres decidiram se automedicar e 6,0\% consultar. Com respeito à prisão de ventre, os homens não tomaram atitude com maior freqüência $(57,1 \%)$, enquanto que as mulheres se automedicaram mais $(57,6 \%)$. Quanto à dor de ouvido, os homens se automedicaram mais $(52,9 \%)$ e as mulheres consultaram mais (18\%).

\section{DISCUSSÃO}

O presente estudo de base populacional, representativo de uma cidade do Sul do Brasil, mostrou quais são os problemas de saúde auto-relatados de maior prevalência. Também mostrou que a maioria desses sintomas foram mais freqüentes nas mulheres, que aumentaram diretamente segundo o grupo de idade e inversamente segundo o grupo socioeconômico e que a

TABELA 2. Prevalência e atitude frente a problemas de saúde auto-relatados segundo sexo, Rio Grande (RS), Brasil, 2000

\begin{tabular}{|c|c|c|c|c|c|c|c|c|c|c|c|c|c|c|}
\hline \multirow[b]{3}{*}{ Sintoma } & \multirow{2}{*}{\multicolumn{4}{|c|}{ Sexo }} & \multicolumn{10}{|c|}{ Comportamento frente ao sintoma (\%) } \\
\hline & & & & & \multicolumn{3}{|c|}{ Não fez nada } & \multicolumn{3}{|c|}{ Automedicação } & \multicolumn{4}{|c|}{ Consulta } \\
\hline & No. & $\mathrm{M}(\%)$ & $F(\%)$ & $P^{a}$ & Total & $M$ & $\mathrm{~F}$ & Total & M & $\mathrm{F}$ & Total & $M$ & $\mathrm{~F}$ & $P^{\mathrm{b}}$ \\
\hline Dor de cabeça & 698 & 36,5 & 63,5 & 0,001 & 31,7 & 32,6 & 31,2 & 64,6 & 63,9 & 65,0 & 3,7 & 3,5 & 3,8 & 0,9 \\
\hline Nervosismo & 618 & 36,1 & 63,9 & 0,001 & 79,9 & 87,9 & 75,4 & 14,6 & 9,4 & 17,5 & 5,5 & 2,7 & 7,1 & 0,001 \\
\hline Dores nas juntas & 448 & 38,2 & 61,8 & 0,001 & 66,5 & 69,6 & 64,6 & 23,9 & 20,5 & 26,0 & 9,6 & 9,9 & 9,4 & 0,4 \\
\hline Dor nas costas & 442 & 39,4 & 60,6 & 0,001 & 73,5 & 71,8 & 74,6 & 20,4 & 23,0 & 18,7 & 6,1 & 5,2 & 6,7 & 0,5 \\
\hline Insônia & 311 & 35,7 & 64,3 & 0,001 & 83,0 & 94,6 & 76,5 & 12,2 & 2,7 & 17,5 & 4,8 & 2,7 & 6,0 & 0,001 \\
\hline Depressão & 280 & 27,7 & 72,5 & 0,001 & 82,9 & 89,6 & 80,3 & 10,0 & 9,1 & 10,3 & 7,1 & 1,3 & 9,4 & 0,06 \\
\hline Prisão de ventre & 268 & 23,1 & 73,9 & 0,001 & 43,3 & 57,1 & 38,4 & 53,0 & 40,0 & 57,6 & 3,7 & 2,9 & 4,0 & 0,03 \\
\hline Dor de garganta & 225 & 43,6 & 56,4 & 0,4 & 40,0 & 32,7 & 45,7 & 50,2 & 55,1 & 46,5 & 9,8 & 12,2 & 7,9 & 0,1 \\
\hline Tosse & 224 & 56,7 & 43,3 & 0,001 & 60,7 & 60,6 & 60,8 & 31,3 & 33,9 & 27,8 & 8,0 & 5,5 & 11,3 & 0,2 \\
\hline Pressão alta & 222 & 32,0 & 68,0 & 0,001 & 39,6 & 39,4 & 39,7 & 35,1 & 40,9 & 32,5 & 25,2 & 19,7 & 27,8 & 0,3 \\
\hline Dor no peito & 209 & 33,5 & 66,5 & 0,001 & 69,4 & 74,3 & 66,9 & 18,2 & 17,1 & 18,7 & 12,4 & 8,6 & 14,4 & 0,4 \\
\hline Falta de ar & 193 & 33,7 & 66,3 & 0,001 & 71,5 & 78,5 & 68,0 & 18,7 & 15,4 & 20,3 & 9,8 & 6,2 & 11,7 & 0,3 \\
\hline Diarréia & 91 & 53,9 & 46,1 & 0,1 & 52,7 & 55,1 & 50,0 & 40,7 & 40,8 & 40,5 & 6,6 & 4,1 & 9,5 & 0,6 \\
\hline Dor de ouvido & 84 & 40,5 & 59,5 & 0,3 & 51,2 & 41,2 & 58,0 & 35,7 & 52,9 & 24,0 & 13,1 & 5,9 & 18,0 & 0,02 \\
\hline Febre & 83 & 44,6 & 55,4 & 0,8 & 26,5 & 27,0 & 26,1 & 51,8 & 51,4 & 52,2 & 21,7 & 21,6 & 21,7 & 0,9 \\
\hline Sangue na urina & 16 & 31,2 & 68,8 & 0,3 & 25,0 & 20,0 & 27,3 & 25,0 & 20,0 & 27,3 & 50,0 & 60,0 & 45,5 & 0,9 \\
\hline Sangue nas fezes & 12 & 33,3 & 66,7 & 0,4 & 50,0 & 50,0 & 50,0 & 33,3 & 25,0 & 37,5 & 16,7 & 25,0 & 12,5 & 0,8 \\
\hline
\end{tabular}

a Teste de associação para distribuição dos sintomas segundo o sexo.

b Teste de independência na distribuição dos sintomas segundo o sexo e o comportamento. 
maior parte das pessoas não tomou nenhuma atitude.

A prevalência geral de problemas de saúde relatados pela população no presente estudo foi de $63 \%$. Um estudo realizado em Botucatu, Brasil, constatou que $56 \%$ das pessoas entrevistadas nos domicílios apresentaram episódios mórbidos nos 30 dias anteriores à entrevista (10). Em nosso estudo, a queixa mais freqüente foi a relacionada à dor em geral, chegando, no caso da cefaléia, a $55 \%$ dos entrevistados, e no caso de dores nas costas e nas juntas, a 35\%, respectivamente. A esse respeito, uma pesquisa realizada na Espanha sobre prevalência de dor na população geral revelou que $43 \%$ dos entrevistados haviam sentido dor na semana anterior (11). Na Noruega, $80 \%$ das pessoas em um estudo referiram problemas osteomusculares (12).

As queixas ou sintomas relacionados à saúde mental foram bem freqüentes entre os entrevistados, mostrando a importância que esses problemas têm para a população. Quarenta e nove por cento das pessoas entrevistadas referiram ter nervosismo, $22 \%$ de pessoas se consideravam portadoras de depressão e 24,7\% apresentaram insônia. Esses valores, apesar de terem sido obtidos por auto-relato, estão bem próximos dos valores citados em outros estudos que utilizaram questionários ou instrumentos específicos. Assim, outro estudo realizado na mesma cidade, que utilizou um instrumento reconhecido, o self reporting questionnaire (SRQ-20), constatou que $13 \%$ dos homens e $22 \%$ das mulheres eram portadores de transtornos psiquiátricos menores (5). Na Cidade de Pelotas (também no Estado do Rio Grande do Sul), um estudo de base populacional com pessoas acima de 20 anos revelou uma prevalência de insônia de 25,2\% no período de 1 mês (13). Ihlebaek et al. (12), na Noruega, encontraram uma prevalência de problemas mentais de $65 \%$.

No presente trabalho, a hipertensão arterial foi informada por $18 \%$ dos entrevistados. Interessantemente, um estudo realizado na Cidade de Pelotas, que realizou a medição da pressão arterial nas pessoas entrevistadas, revelou que perto de $20 \%$ das pessoas tinham cifras tensionais elevadas (14). Pode-se notar, assim, uma aproximação entre algumas das prevalências referidas e as prevalências obtidas mediante instrumentos específicos.

Observamos que o risco de apresentar problemas de saúde aumentou conforme diminuiu a classe social. As RP calculadas para os problemas de saúde referidos, ajustadas por sexo, grupo de idade e grupo socioeconômico, mostraram como a prevalência dos sintomas referidos aumenta linearmente quando se passa de um grupo a outro. Isso evidencia que os mais pobres apresentaram uma carga maior de morbidade referida. Nos grupos mais pobres, os sintomas relacionados à saúde mental, às dores osteomusculares e às doenças crônicas (tosse, falta de ar, pressão alta) tiveram as maiores RP. De forma semelhante, um estudo que avaliou a prevalência de morbidade e o nível de escolaridade em 11 países diferentes também encontrou que a prevalência da morbidade estava associada à variável socioeconômica (15). Um outro estudo que avaliou a saúde mental em alguns países mostrou a existência de uma associação inversa entre nível socioeconômico e transtornos mentais comuns (16).

No que se refere ao sexo, o risco de apresentar algum dos sintomas pesquisados foi sempre mais alto entre as mulheres, com exceção da tosse, possivelmente pelo fato de o tabagismo estar associado aos homens (17). É provável que isso esteja relacionado à tendência das mulheres de recordar ou informar melhor do que os homens sobre sua história mórbida (18).

Com respeito à idade, o tipo de sintoma determinou se a tendência linear foi ascendente ou descendente. Sintomas como febre, dor de garganta e dor de ouvido apresentaram uma relação dose-resposta inversa segundo o grupo de idade, por serem mais freqüentemente associados a doenças que afetam faixas mais jovens. Já os sintomas relacionados às doenças crônicas, como dores articulares, falta de ar e pressão alta, que afetam os mais idosos, aumentaram linearmente com a idade.

Outro aspecto interessante do estudo refere-se à análise da atitude das pessoas frente ao sintoma apresentado. Foi possível verificar o fenômeno conhecido como iceberg da morbidade, já referido por Last (19), uma vez que apenas $8,2 \%$ dos problemas relatados pelas pessoas entrevistadas resultaram em consulta médica. A grande maioria dos problemas de saúde informados $(60,2 \%)$ não geraram nenhum tipo de cuidado por parte do indivíduo, e 32\% preferiram a automedicação.

A análise do comportamento frente ao sintoma segundo sexo foi muito semelhante entre homens e mulheres, e só para alguns sintomas houve predominância feminina. Isso ocorreu com sintomas relacionados à saúde mental, como nervosismo e depressão, mas também com insônia, prisão de ventre e dor de ouvido. Essa diferença vai ao encontro do maior uso dos serviços de saúde por parte das mulheres (20). Porém, somente em quatro situações houve diferença significativa na forma de reagir ao problema de saúde: nervosismo, insônia, prisão de ventre e dor de ouvido. No caso do nervosismo, da insônia e da prisão de ventre as mulheres se automedicaram mais e consultaram mais. Já no caso dos homens, o mais freqüente foi não fazer nada diante desses sintomas. A falta de diferença no comportamento entre sexos para algum dos sintomas pode ter resultado da falta de poder estatístico, como no caso de sangue nas fezes, sangue na urina, dor de ouvido e febre.

Tais resultados também trazem à tona a questão da carga de morbidade em excesso apresentada pelas mulheres e do comportamento diferente apresentado quando comparadas aos homens, como se observou para alguns dos sintomas pesquisados neste estudo. Essa diferença poderia ser atribuída a fatores tanto biológicos quanto psicossociais $(18,21)$. O papel social da mulher no trabalho e na família influencia a morbidade (21). O acúmulo de um emprego além do cuidado com os filhos poderia afetar a carga de morbidade. Também existe uma predispo- 
sição maior da mulher em recordar melhor os problemas de saúde, assim como de informar melhor os sintomas que apresenta (18). Contudo, as variações em determinados casos podem ser a favor dos homens, ou simplesmente pode não haver diferença alguma, dependendo do tipo de morbidade estudada (22).

Em conclusão, o estudo permitiu evidenciar que apenas uma pequena parcela das pessoas com problemas de saúde busca uma consulta médica e que as classes mais pobres apresentam uma proporção maior de determinados sintomas relacionados com a saúde mental, com o trabalho físico e com a falta de cuidados em saúde. Mostrou, também, que é necessário estabelecer e reforçar políticas de saúde pública que enfatizem a questão da saúde mental e a educação para o auto-cuidado, salientando quais sinais e sintomas indicam a necessidade de procurar um serviço de saúde. É preciso estudar mais profundamente o papel da classe social na procura de cuidados e na determinação dos comportamentos em saúde. Finalmente, o estudo proporcionou subsídios aos planejadores em saúde para uma alocação mais direcionada dos recursos, e serve aos educadores em saúde como estímulo para desenvolver intervenções direcionadas à busca mais adequada dos serviços de saúde.

\section{REFERÊNCIAS}

1. Heistaro S, Jousilahti $P$, Lahelma E, Vartiainen E, Puska P. Self rated health and mortality: a long term prospective study in eastern Finland. J Epidemiol Community Health. 2001; 55(4):227-32.

2. Reijneveld SA, Stronks K. The validity of selfreported use of health care across socioeconomic strata: a comparison of survey and registration data. Int J Epidemiol. 2001;30(6): 1407-14.

3. Mendoza-Sassi R, Béria JU. Utilización de los servicios de salud: una revisión sistemática sobre los factores relacionados. Cad Saude Publ. 2001;17(Supl):819-32.

4. Instituto Brasileiro de Geografia e Estatística. Pesquisa Nacional por Amostragem de Domicílios (PNAD). Acesso e utilização de serviços de saúde. Brasília; IBGE: 1998.

5. Mendoza-Sassi R, Beria JU, Barros AJ. Outpatient health service utilization and associated factors: a population-based study. Rev Saude Publica. 2003;37(3):372-8.

6. Pinheiro R, Viacava F, Travassos C, Brito A. Gênero, morbidade, acesso e utilizaçäo de serviços de saúde no Brasil. Cienc Saude Coletiva. 2002;7(4):687-707.

7. Instituto Brasileiro de Geografia e Estatística. Censo demográfico de 2000. Disponível em: www.ibge.gov.br. Acessado abril de 2005.

8. ANEP. Economic classification criterionBrazil. Disponível em: www.anep.org.br/ CCEB_ENGLISH.pdf. Acessado maio de 2005.
9. Fraser C. Setting the scene. Em: Fraser RC, editor. Clinical method. A general practice approach. $2^{\mathrm{a}}$ ed. Cambridge: Butterworth Heinemann; 1992. Pp. 1-24.

10. Lebrão M, Carandina L, Magaldi C. Análise das condições de saúde e de vida da população urbana de Botucatu, São Paulo (Brasil). IV-Morbidade referida em entrevistas domiciliárias, 1983-1984. Rev Saude Publ. 1991; 25(6):425-60.

11. Catala E, Reig E, Artes M, Aliaga L, López JS, Segu JL. Prevalence of pain in the Spanish population: telephone survey in 5000 homes. Eur J Pain. 2002;6(2):133-40.

12. Ihlebaek C, Eriksen HR, Ursin H. Prevalence of subjective health complaints (SHC) in Norway. Scand J Public Health. 2002;30(1):20-9.

13. Oliveira AVG. Prevalência de insônia e fatores associados na população adulta da cidade de Pelotas, RS [dissertação de mestrado]. Pelotas: UFPEL; 2000

14. Piccini R, Victora C. Hipertensão arterial sistêmica em área urbana no sul do Brasil: prevalência e fatores de risco. Rev Saude Publ. 1994;28(4):261-7.

15. Cavelaars AE, Kunst AE, Geurts JJ, Crialesi R, Grotvedt L, Helmert U, et al. Differences in self reported morbidity by educational level: a comparison of 11 western European countries. J Epidemiol Community Health. 1998; 52(4):219-27.
16. Patel V, Araya R, de Lima M, Ludermir A Todd C. Women, poverty and common mental disorders in four restructuring societies. Soc Sci Med. 1999;49(11):1461-71.

17. Moreira L, Fuchs F, Moraes R, Bredemeier M, Cardozo S. Prevalência de tabagismo e fatores associados em área metropolitana da região Sul do Brasil. Rev Saude Publ. 1995;29(1): 46-51.

18. Verbrugge LM. Gender and health: an update on hypotheses and evidence. J Health Soc Behav. 1985;26(3):156-82.

19. Last J. The iceberg: completing the clinical picture in general practice. Lancet. 1963;2:28-31.

20. Travassos C, Viacava F, Pinheiro R, Brito A Utilização dos serviços de saúde no Brasil: gênero, características familiares e condição social. Rev Panam Salud Publica. 2002;11(5/6): 365-73.

21. Hibbard JH, Pope CR. The quality of social roles as predictors of morbidity and mortality. Soc Sci Med. 1993;36(3):217-25.

22. Macintyre S, Hunt K, Sweeting H. Gender differences in health: are things really as simple as they seem? Soc Sci Med. 1996;42(4):617-24.

Manuscrito recebido em 29 de junho de 2005. Aceito em versão revisada em 11 de maio de 2006. 
ABSTRACT Objective. To determine the prevalence of signs and symptoms in the adult population, the sociodemographic factors associated with them, and the actions taken as a result of these symptoms, according to sex.

Prevalence of signs and symptoms, associated sociodemographic factors and resulting actions in an urban center in southern Brazil

Key words
Methods. A population-based cross-sectional study was carried out in the city of Rio Grande (state of Rio Grande do Sul), Brazil, in 2000. We interviewed 1259 people $\geq 15$ years of age. Data were collected using a structured questionnaire containing 18 symptoms in addition to sociodemographic questions. The prevalence of symptoms and prevalence ratios for sex, age, and socioeconomic status were estimated after alternately adjusting for these variables. The actions resulting from the presence of symptoms were also analyzed for each sex.

Results. Mean age was 40.33 years (53.9\% were women). The most prevalent symptom was headache $(55.4 \%)$. Joint pain, insomnia, constipation, high blood pressure, and shortness of breath increased with age. The following were more prevalent among the lower social classes: headache, nervousness, joint and back pain, insomnia and depression, high blood pressure, chest pain, and shortness of breath. A total of 4424 health problems were reported (an average of 3.25 per person); $60.2 \%$ did not generate any action, $31.6 \%$ resulted in self-medication, and $8.2 \%$ resulted in a visit to a medical facility. Headache, nervousness, joint and back pain, insomnia, depression, constipation, high blood pressure, chest pain, and shortness of breath were significantly higher in women, whereas cough was significantly more prevalent in men. Women and men took similar actions in the presence of signs and symptoms.

Conclusion. It is necessary to establish health policies that lay an emphasis on mental health and education for self care and on those symptoms that signal the need to go to a health facility. It is also necessary to study the role of social class in determining health behavior and the choice to seek care.

Social class, patient acceptance of health care, prevalence, signs and symptoms, Brazil.

\section{Mantenerse en forma para la vida. Necesidades nutricionales de los adultos mayores}

La Organización Panamericana de la Salud (OPS) reconoce que proteger la salud de los adultos mayores es uno de los principales desafíos que enfrenta la salud pública en el siglo XXI. Una buena alimentación y el mantenerse en forma son maneras de garantizar la salud y el bienestar en la vejez. En este marco, la OPS se complace en presentar su más reciente publicación, Mantenerse en forma para la vida: necesidades nutricionales de los adultos mayores, obra que representa una fuente autorizada de información sobre la alimentación y actividad física recomendadas para los adultos mayores. En el cuerpo principal de la obra se discuten los aspectos epidemiológicos y sociales del envejecimiento, los cambios funcionales y de salud que se producen al envecejer, los efectos de la actividad física, la evaluación del estado nutricional de los adultos mayores y las pautas nutricionales que deben observarse para envejecer sanamente.

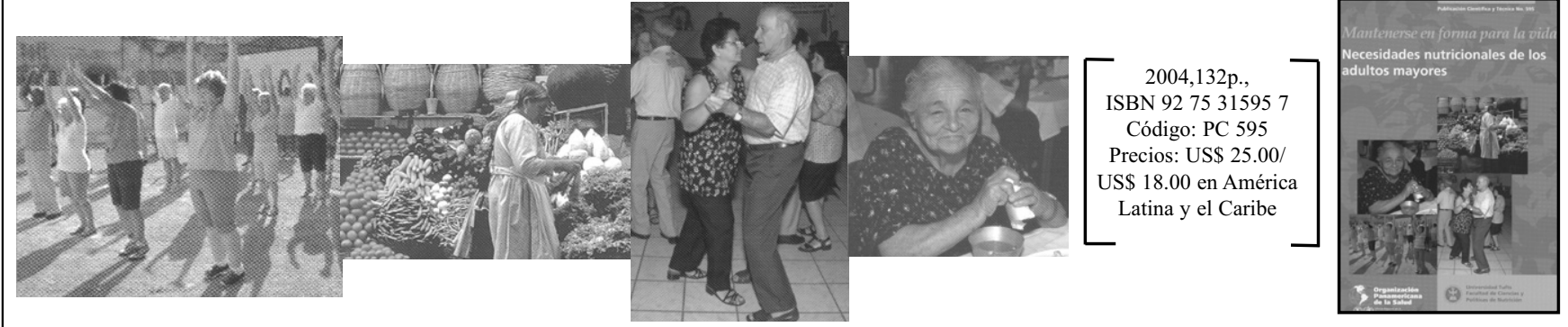

Esta publicación será de mucha utilidad para nutricionistas, médicos generales, gerontólogos, personal de enfermería, proveedores de atención, formuladores de políticas públicas, salubristas y trabajadores sociales. Las recomendaciones específicas sobre la ingesta de nutrientes, el ejercicio y la actividad física también deben interesar a los lectores en general.

Adquiera esta publicación por medio de la librería en línea de la OPS: http://publications.paho.org; correo electrónico: paho@pmds.com 